International Journal of Pure and Applied Mathematics

Volume 92 No. 3 2014, 369-379

ISSN: 1311-8080 (printed version); ISSN: 1314-3395 (on-line version)

url: http://www.ijpam.eu

doi: http://dx.doi.org/10.12732/ijpam.v92i3.5

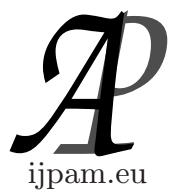

\title{
ON $Q$-FUZZY IDEALS IN ORDERED SEMIGROUPS
}

\author{
Somsak Lekkoksung \\ Department of Mathematics \\ Faculty of Engineering \\ Rajamangala University of Technology Isan \\ Khon Kaen Campus, THAILAND
}

\begin{abstract}
In this paper we shows that in ordered groupoids the $Q$-fuzzy right (resp. $Q$-fuzzy left) ideals are $Q$-fuzzy quasi-ideals, in ordered semigroups the $Q$-fuzzy quasi-ideals are $Q$-fuzzy bi-ideals, and in regular ordered semigroups the $Q$-fuzzy quasi-ideals and the $Q$-fuzzy bi-ideals coincide and show that if $S$ is an ordered semigroup, then a $Q$-fuzzy subset $f$ is a $Q$-fuzzy quasi-ideal of $S$ if and only if there exist a $Q$-fuzzy right ideal $g$ and a $Q$-fuzzy left ideal $h$ of $S$ such that $f=g \cap h$.
\end{abstract}

AMS Subject Classification: 06F05

Key Words: ordered semigroup, regular ordered semigroup, $Q$-fuzzy left (right) ideal, $Q$-fuzzy quasi-ideal, $Q$-fuzzy bi-ideals

\section{Introduction}

A fuzzy set theory was conceptualized by Professor L. A. Zadeh at the University of California in 1965, [14] as a generalization of abstract set theory. Zadehs initiation is virtually a complete paradigm shift that initially gained popularity in the Far East and its successful applications has gained further ground almost round the globe. Rosenfeld [11] used the ideal of fuzzy set to introduce the no-

Received: October 19, 2013

(c) 2014 Academic Publications, Ltd. url: www.acadpubl.eu 
tions of fuzzy subgroups. The ideal of fuzzy subsemigroup was also introduced by Kuroki [7], [9]. In [8], Kuroki characterized several classes of semigroups in terms of fuzzy left, fuzzy right and fuzzy bi-ideals. Xie [12] introduced the idea of extensions of fuzzy ideals in semigroups. The concept of fuzzy generalized bi-ideals of an ordered semigroup is introduced by Xie and Tang [13] and characteried regular ordered semigroups by means of fuzzy left ideals, fuzzy right ideals and fuzzy (generalized) bi-ideals. In [10], Majumder introduced the concept of $Q$-fuzzification of ideals of $\Gamma$-semigroups and some important properties have been investigated. In this paper we shows that in ordered groupoids the $Q$-fuzzy right (resp. $Q$-fuzzy left) ideals are $Q$-fuzzy quasi-ideals, in ordered semigroups the $Q$-fuzzy quasi-ideals are $Q$-fuzzy bi-ideals, and in regular ordered semigroups the $Q$-fuzzy quasi-ideals and the $Q$-fuzzy bi-ideals coincide and show that if $S$ is an ordered semigroup, then a $Q$-fuzzy subset $f$ is a $Q$ fuzzy quasi-ideal of $S$ if and only if there exist a $Q$-fuzzy right ideal $g$ and a $Q$-fuzzy left ideal $h$ of $S$ such that $f=g \cap h$.

\section{Preliminaries}

Throughout this paper, unless stated otherwise, $S$ stands for an ordered semigroup. A function $f$ from $S \times Q$ to the real closed interval [0,1] is called $Q$-fuzzy subset of $S$, where $Q$ is a non-empty set. The ordered semigroup $S$ itself is a $Q$-fuzzy subset of $S$, its characteristic function, also denoted by $S$, is defined as follows:

$$
S: S \times Q \longrightarrow[0,1] \mid(x, q) \mapsto S(x, q):=1,
$$

for all $x \in S$ and $q \in Q$.

Let $f$ and $g$ be two $Q$-fuzzy subsets of $S$. Then the inclusion relation $f \subseteq g$ means that

$$
f(x, q) \leq g(x, q),
$$

for all $x \in S$ and $q \in Q, f \cap g$ and $f \cup g$ are defined by

$$
\begin{aligned}
& (f \cap g)(x, q):=\min \{f(x, q), g(x, q)\}, \\
& (f \cup g)(x, q):=\max \{f(x, q), g(x, q)\},
\end{aligned}
$$

for all $x \in S$ and $q \in Q$.

Let $S$ and $Q$ be an ordered semigroup and a non-empty set, respectively. For $x \in S$, we define $A_{x}:=\{(y, z) \in S \times S \mid x \leq y z\}$. The product $f \circ g$ of $f$ 
and $g$ is defined by

$$
(\forall x \in S, \forall q \in Q)(f \circ g)(x, q):=\left\{\begin{array}{cc}
\bigvee_{(y, z) \in A_{x}} \min \{f(y, q), g(z, q)\} & \text { if } A_{x} \neq \emptyset, \\
0 & \text { if } A_{x}=\emptyset,
\end{array}\right.
$$

We denote by $f_{A \times Q}$ the characteristic function of $A \times Q$, that is, the mapping of $S \times Q$ into $[0,1]$ defined by

$$
f_{A \times Q}(x, q):= \begin{cases}1 & \text { if } x \in A \times Q \\ 0 & \text { if } x \notin A \times Q\end{cases}
$$

for all $(x, q) \in A \times Q$.

\section{Main Results}

In this section, we introduced the notion of $Q$-fuzzy right (resp. $Q$-fuzzy left) ideals, $Q$-fuzzy quasi-ideals, $Q$-fuzzy bi-ideals of ordered semigroups, and investigate related properties.

Definition 3.1. Let $S$ and $Q$ be an ordered groupoid and a non-empty set, respectively. A $Q$-fuzzy subset $f$ of $S$ is called a $Q$-fuzzy right ideal (resp. $Q$-fuzzy left) ideal of $S$ if:

(1) $x \leq y$ implies $f(x, q) \geq f(y, q)$, and

(2) $f(x y, q) \geq f(x, q)($ resp. $f(x y, q) \geq f(y, q))$,

for all $x, y \in S$ and for all $q \in Q$.

Definition 3.2. Let $S$ and $Q$ be an ordered groupoid and a non-empty set, respectively. A $Q$-fuzzy subset $f$ of $S$ is called a $Q$-fuzzy quasi-ideal of $S$ if:

(1) $x \leq y \Rightarrow f(x, q) \geq f(y, q)$,

(2) $(f \circ S) \cap(S \circ f) \subseteq f$,

for all $x, y \in S$ and for all $q \in Q$.

Definition 3.3. Let $S$ and $Q$ be an ordered semigroup and a non-empty set, respectively. A $Q$-fuzzy subsemigroup $f$ of $S$ is called a $Q$-fuzzy bi-ideal of $S$ if:

(1) $x \leq y \Rightarrow f(x, q) \geq f(y, q)$,

(2) $f(x y z, q) \geq \min \{f(x, q), f(z, q)\}$,

for all $x, y, z \in S$ and for all $q \in Q$. 
Theorem 3.4. If $S$ is an ordered groupoid and $Q$ is a non-empty set, then the $Q$-fuzzy right (resp. left) ideals of $S$ are $Q$-fuzzy quasi-ideals of $S$.

Proof. Let $f$ be a $Q$-fuzzy right ideal of $S$ and $x \in S, q \in Q$. First of all,

$$
((f \circ S) \cap(S \circ f))(x, q)=\min \{(f \circ S)(x, q),(S \circ f)(x, q)\} .
$$

If $A_{x}=\emptyset$, then we have $(f \circ S)(x, q)=0=(S \circ f)(x, q)$ and, since $f$ is a $Q$-fuzzy right ideal of $S$, we have $\min \{(f \circ S)(x, q),(S \circ f)(x, q)\}=0 \leq f(x, q)$.

If $A_{x} \neq \emptyset$, then

$$
(f \circ S)(x, q)=\bigvee_{(u, v) \in A_{x}}\{\min \{f(u, q), S(v, q)\}\} .
$$

On the other hand, if $(u, v) \in A_{x}$, then $x \leq u v$ and $f(x, q) \geq f(u v, q) \geq$ $f(u, q)=\min \{f(u, q), S(v, q)\}$. Hence, we have

$$
\begin{aligned}
f(x, q) & \geq \bigvee_{(u, v) \in A_{x}}\{\min \{f(u, q)\}\} \\
& \geq \min \{(f \circ S)(x, q),(S \circ f)(x, q)\} \\
& =((f \circ S) \cap(S \circ f))(x, q) .
\end{aligned}
$$

Therefore $f$ is a $Q$-fuzzy quasi-ideal of $S$.

Theorem 3.5. If $S$ is an ordered semigroup and $Q$ is a non-empty set, then the $Q$-fuzzy quasi-ideals are $Q$-fuzzy bi-ideals of $S$.

Proof. Let $f$ be a $Q$-fuzzy quasi-ideal of $S$ and $x, y, z \in S, q \in Q$. Then we have

$$
f(x y z, q) \geq((f \circ S) \cap(S \circ f))(x y z, q)=\min \{(f \circ S)(x y z, q),(S \circ f)(x y z, q)\} .
$$

Since $(x, y z) \in A_{x y z}$, we have

$$
\begin{aligned}
(f \circ S)(x y z, q) & =\bigvee_{(u, v) \in A_{x y z}}\{\min \{f(u, q), S(v, q)\}\} \\
& \geq \min \{f(x, q), S(y z, q)\} \\
& =f(x, q) .
\end{aligned}
$$

Since $(x y, z) \in A_{x y z}$, we have

$$
(S \circ f)(x y z, q)=\bigvee_{(u, v) \in A_{x y z}}\{\min \{S(u, q), f(v, q)\}\}
$$




$$
\begin{aligned}
& \geq \min \{S(x y, q), f(z, q)\} \\
& =f(z, q) .
\end{aligned}
$$

Thus we have

$$
f(x y z, q) \geq \min \{(f \circ S)(x y z, q),(S \circ f)(x y z, q)\} \geq \min \{f(x, q), f(z, q)\} .
$$

Hence $f$ is a $Q$-fuzzy bi-ideal of $S$.

An ordered semigroup $S$ is called regular if for any $a \in S$ there exists $x \in S$ such that $a \leq a x a$.

Theorem 3.6. If $S$ is a regular ordered semigroup and $Q$ is a non-empty set, then the $Q$-fuzzy quasi-ideals and the $Q$-fuzzy bi-ideals coincide.

Proof. Let $f$ be a $Q$-fuzzy bi-ideal of $S$ and $x \in S, q \in Q$. We will prove that

$$
((f \circ S) \cap(S \circ f))(x, q) \leq f(x, q)
$$

First of all, we have

$$
((f \circ S) \cap(S \circ f))(x, q)=\min \{(f \circ S)(x, q),(S \circ f)(x, q)\} .
$$

If $A_{x}=\emptyset$, then as we have already seen in Theorem 3.4, condition (1) is satisfied.

If $A_{x} \neq \emptyset$, then

$$
\begin{aligned}
& (f \circ S)(x, q)=\bigvee_{(z, w) \in A_{x}}\{\min \{f(z, q), S(w, q)\}\}, \\
& (S \circ f)(x, q)=\bigvee_{(u, v) \in A_{x}}\{\min \{S(u, q), f(v, q)\}\} .
\end{aligned}
$$

Let $(f \circ S)(x, q) \leq f(x, q)$. Then, we have

$$
\begin{aligned}
f(x, q) & \geq(f \circ S)(x, q) \\
& \geq \min \{(f \circ S)(x, q),(S \circ f)(x, q)\} \\
& =((f \circ S) \cap(S \circ f))(x, q),
\end{aligned}
$$

and condition (1) is satisfied.

Let $(f \circ S)(x, q)>f(x, q)$. Then, by (2), there exists $(z, w) \in A_{x}$ such that

$$
\min \{f(z, q), S(w, q)\}>f(x, q)
$$


(otherwise $f(x, q) \leq(f \circ S)(x, q)$, which is impossible). Since $(z, w) \in A_{x}$, we have $z, w \in S$ and $x \leq z w$. Similarly, from $\min \{f(z, q), S(w, q)\}=f(z, q)$, by (4), we obtain

$$
f(z, q)>f(x, q) .
$$

We will prove that $(S \circ f)(x, q) \leq f(x, q)$, then

$$
\min \{(f \circ S)(x, q),(S \circ f)(x, q)\} \leq(S \circ f)(x, q) \leq f(x, q),
$$

so that $((f \circ S) \cap(S \circ f))(x, q) \leq f(x, q)$, and condition (1) is satisfied.

By (3), it is enough to prove that

$$
\min \{S(u, q), f(v, q)\} \leq f(x, q), \forall(u, v) \in A_{x} .
$$

Let $(u, v) \in A_{x}$. Then $x \leq u v$ for some $u, v \in S$. Since $S$ is regular, there exists $s \in S$ such that $x \leq x s x$. It follows that $x \leq z w s u v$. Since $f$ is a $Q$-fuzzy bi-ideal of $S$, we have

$$
\min \{S(u, q), f(v, q)\} \leq f(x, q), \forall(u, v) \in A_{x}
$$

and, we have

$$
f(x, q) \geq f(z w s u v, q) \geq \min \{f(z, q), f(v, q)\} .
$$

If $\min \{f(z, q), f(v, q)\}=f(z, q)$, then $f(z, q) \leq f(x, q)$ which is impossible by (5). Thus we have $\min \{f(z, q), f(v, q)\}=f(v, q)$, then $f(x, q) \geq f(v, q)=$ $\min \{S(u, q), f(v, q)\}$.

In the following, using the usual definitions of ideals mentioned above, we show that the $Q$-fuzzy quasi-ideals of an ordered semigroup are just intersections of $Q$-fuzzy right and $Q$-fuzzy left ideals.

Lemma 3.7. Let $S$ and $Q$ be an ordered semigroup and a non-empty set respectively. Let $f$ be a $Q$-fuzzy subset of $S$. Then we have the following:

(1) $(S \circ f)(x y, q) \geq f(y, q)$ for all $x, y \in S, q \in Q$,

(2) $(S \circ f)(x y, q) \geq(S \circ f)(y, q)$ for all $x, y \in S, q \in Q$.

Proof. (1) Let $x, y \in S$ and $q \in Q$. Since $(x, y) \in A_{x y}$, we have

$$
(S \circ f)(x y, q)=\bigvee_{(w, z) \in A_{x y}}\{\min \{S(w, q), f(z, q)\}\} \geq \min \{S(x, q), f(y, q)\}=f(y, q) .
$$


(2) Let $x, y \in S$ and $q \in Q$. If $A_{y}=\emptyset$, then $(S \circ f)(y, q)=0$. Since $(S \circ f)$ is a $Q$-fuzzy subset of $S$, we have $(S \circ f)(x y, q) \geq 0=(S \circ f)(y, q)$. If $A_{x} \neq \emptyset$, then

$$
(S \circ f)(y, q)=\bigvee_{(w, z) \in A_{y}}\{\min \{S(w, q), f(z, q)\}\} .
$$

On the other hand,

$$
(S \circ f)(x y, q) \geq \min \{S(w, q), f(z, q)\}, \forall(w, z) \in A_{y} .
$$

Indeed, let $(w, z) \in A_{y}$. Since $(x, y) \in A_{x y}$, we have

$$
(S \circ f)(x y, q)=\bigvee_{(s, t) \in A_{x y}}\{\min \{S(s, q), f(t, q)\}\} .
$$

Since $(w, z) \in A_{y}$, we have $y \leq w z$, then $x y \leq x w z$, and $(x w, z) \in A_{x y}$. Hence we have

$$
(S \circ f)(x y, q) \geq \min \{S(x w, q), f(z, q)\}=f(z, q)=\min \{S(w, q), f(z, q)\} .
$$

By (6), we have

$$
(S \circ f)(x y, q) \geq \bigvee_{(w, z) \in A_{y}}\{\min \{S(w, q), f(z, q)\}\}=(S \circ f)(y, q) .
$$

In a similar way we can prove the following lemmas:

Lemma 3.8. Let $S$ and $Q$ be an ordered semigroup and a non-empty set, respectively. Let $f$ be a $Q$-fuzzy subset of $S$. Then we have the following:

(1) $(S \circ f)(x y, q) \geq f(x, q)$ for all $x, y \in S, q \in Q$,

(2) $(S \circ f)(x y, q) \geq(S \circ f)(x, q)$ for all $x, y \in S, q \in Q$.

Lemma 3.9. Let $S$ and $Q$ be an ordered semigroup and a non-empty set, respectively. Let $f$ be a $Q$-fuzzy subset of $S$ and $x \leq y$. Then we have $(S \circ f)(x, q) \geq(S \circ f)(y, q)$, for all $q \in Q$.

Proof. Let $x, y \in S$ and $q \in Q$. Then, if $A_{y}=\emptyset$, then $(S \circ f)(y, q)=0$. Since $S \circ f$ is a $Q$-fuzzy subset of $S$, we have $(S \circ f)(x, q) \geq 0$, then $(S \circ f)(x, q) \geq$ $(S \circ f)(y, q)$. If $A_{y} \neq \emptyset$, then

$$
(S \circ f)(y, q)=\bigvee_{(w, z) \in A_{y}}\{\min \{S(w, q), f(z, q)\}\}=\bigvee_{(w, z) \in A_{y}}\{f(z, q)\}
$$


On the other hand,

$$
(S \circ f)(x, q) \geq f(z, q), \forall(w, z) \in A_{y} .
$$

Indeed, let $(w, z) \in A_{y}$. Since $x \leq y \leq w z$, we have $(w, z) \in A_{x}$. Then

$$
(S \circ f)(x y, q)=\bigvee_{(s, t) \in A_{x y}}\{\min \{S(s, q), f(t, q)\}\} \geq \min \{S(w, q), f(z, q)\}=f(z, q)
$$

Thus, by (7), we have

$$
(S \circ f)(x, q) \geq \bigvee_{(w, z) \in A_{y}}\{f(z, q)\}=(S \circ f)(y, q)
$$

The proof is completed.

Lemma 3.10. Let $S$ and $Q$ be an ordered semigroup and a non-empty set, respectively. Let $f$ be a $Q$-fuzzy subset of $S$ and $x \leq y$. Then we have $(f \circ S)(x, q) \geq(f \circ S)(y, q)$, for all $q \in Q$.

Lemma 3.11. Let $S$ and $Q$ be an ordered semigroup and a non-empty set, respectively. Let $f$ be a $Q$-fuzzy subset of $S$ such that $x \leq y$, we have $f(x, q) \geq f(y, q)$ for all $x, y \in S, q \in Q$. Then the $Q$-fuzzy subset $f \cup(S \circ f)$ is a $Q$-fuzzy left ideal of $S$.

Proof. Let $x, y \in S$ and $q \in Q$. By, Theorem 3.9, we have $(f \cup(S \circ$ $f))(x y, q) \geq(f \cup(S \circ f))(y, q)$. Let now $x \leq y$. Then $(f \cup(S \circ f))(x, q) \geq$ $(f \cup(S \circ f))(y, q)$. Indeed: Since $f$ is a $Q$-fuzzy subset of $S$ and $x \leq y$, by Lemma 3.7 , we get $(S \circ f)(x, q) \geq(S \circ f)(y, q)$ and, by hypothesis, $f(x, q) \geq f(y, q)$. Then

$$
\begin{aligned}
(f \cup(S \circ f))(x, q) & =\max \{f(x, q),(S \circ f)(x, q)\} \\
& \geq \max \{f(y, q),(S \circ f)(y, q)\} \\
& =(f \cup(S \circ f))(y, q) .
\end{aligned}
$$

In a similar way we can prove the following:

Lemma 3.12. Let $S$ and $Q$ be an ordered semigroup and a non-empty set, respectively. Let $f$ be a $Q$-fuzzy subset of $S$ such that $x \leq y$, we have $f(x, q) \geq f(y, q)$ for all $x, y \in S, q \in Q$. Then the $Q$-fuzzy subset $f \cup(f \circ S)$ is a $Q$-fuzzy right ideal of $S$. 
Lemma 3.13. Let $S$ and $f, g, h$ be an ordered semigroup and $Q$-fuzzy subsets of $S$, respectively. Then

$$
f \cap(g \cup h)=(f \cap g) \cup(f \cap h) .
$$

Proof. Let $x \in S$ and $q \in Q$. Then we have

$$
\begin{aligned}
(f \cap(g \cup h))(x, q) & =\min \{f(x, q),(g \cup h)(x, q)\} \\
& =\min \{f(x, q), \max \{g(x, q), h(x, q)\}\} \\
& =\max \{\min \{f(x, q), g(x, q)\}, \min \{f(x, q), h(x, q)\} \\
& =\max \{(f \cap g)(x, q),(f \cap h)(x, q)\} \\
& =((f \cap g) \cup(f \cap h))(x, q) .
\end{aligned}
$$

Corollary 3.14. . Let $S$ and $Q$ be an ordered semigroup. Then the set of all $Q$-fuzzy subsets of $S$ is a distributive lattice.

Theorem 3.15. Let $S$ and $Q$ be an ordered semigroup and a non-empty set, respectively. Then a $Q$-fuzzy subset $f$ of $S$ is a $Q$-fuzzy quasi-ideal of $S$ if and only if there exist a $Q$-fuzzy right ideal $g$ and a $Q$-fuzzy left ideal $h$ of $S$ such that $f=g \cap h$.

Proof. $(\Rightarrow)$. By Lemma 3.11 and Lemma 3.12, $f \cup(S \circ f)$ is a $Q$-fuzzy left ideal and $f \cup(f \circ S)$ is a $Q$-fuzzy right ideal of $S$. Moreover, we have

$$
f=(f \cup(S \circ f)) \cap(f \cup(f \circ S)) .
$$

In fact, by Corolary 3.14, we have

$$
\begin{aligned}
(f \cup(S \circ f)) \cap(f \cup(f \circ S))= & ((f \cup(S \circ f)) \cap f) \cup((f \cup(S \circ f)) \cap(f \circ S)) \\
= & (f \cap f) \cup((S \circ f) \cap f) \cup(f \cap(f \circ S)) \cup((S \circ f) \\
& \cap(f \circ S)) \\
= & f \cup((S \circ f) \cap f) \cup(f \cap(f \circ S)) \cup((S \circ f) \\
& \cap(f \circ S)) .
\end{aligned}
$$

Since $f$ is a $Q$-fuzzy quasi-ideal of $S$, we have $(f \circ S) \cap(S \circ f) \subseteq f$. Besides, $(S \circ f) \cap f \subseteq f$ and $f \cap(f \circ S) \subseteq f$. Hence

$$
(f \cup(S \circ f)) \cap(f \cup(f \circ S))=f .
$$

$(\Leftarrow)$. Let $x \in S$ and $q \in Q$. Then

$$
((f \circ S) \cap(S \circ f))(x, q) \leq f(x, q)
$$


In fact, $((f \circ S) \cap(S \circ f))(x, q)=\min \{(f \circ S)(x, q),(S \circ f)(x, q)\}$. If $A_{x}=\emptyset$, then $(f \circ S)(x, q)=0=(S \circ f)(x, q)$. Thus, in this case condition (8) is satisfied. If $A_{x} \neq \emptyset$, then

$$
(f \circ S)(x, q)=\bigvee_{(y, z) \in A_{x}}\{\min \{f(y, q), S(z, q)\}\}=\bigvee_{(y, z) \in A_{x}}\{f(y, q)\}
$$

We have

$$
f(y, q) \leq h(x, q), \forall(y, z) \in A_{x} .
$$

Indeed, for $(y, z) \in A_{x}$, we have $x \leq y z$ and $h(x, q) \geq h(y z, q) \geq h(y, q)$ because $h$ is a $Q$-fuzzy left ideal of $S$. Thus, applying (10) to (9), we obtain

$$
(f \circ S)(x, q)=\bigvee_{(y, z) \in A_{x}}\{f(y, q)\} \leq h(x, q)
$$

In a similar way, we get $(S \circ f)(x, q) \leq g(x, q)$. Hence

$$
\begin{aligned}
((f \circ S) \cap(S \circ f))(x, q) & =\min \{(f \circ S)(x, q),(S \circ f)(x, q)\} \\
& \leq \min \{h(x, q), g(x q)\} \\
& =(h \cap g)(x, q) \\
& =f(x, q),
\end{aligned}
$$

which completes the proof of (8).

\section{Acknowledgments}

This research is supported by Thailand Research Fund under Grant: MRG5580042.

\section{References}

[1] P. Dheena, S. Coumaressane, Characterization of regular $\Gamma$-semigroups through fuzzy ideals, Iranian Journal of Fuzzy Systems, 4, No. 2 (2007), 57-68.

[2] K. Iseki, A characterization of regular semigroups, Proc. Japan Acad., 32 (1956), 676-677.

[3] N. Kehayopulu, On regular, intra-regular ordered semigroups, Pure Math, and Appl., 4, No. 4 (1993), 447-461. 
[4] N. Kehayopulu, M. Tsingelis, A note on fuzzy sets in semigroups, Sci. Math., 2, No. 3 (1999), 411-413.

[5] N. Kehayopulu, M. Tsingelis, Fuzzy sets in ordered groupoids, Semigroup Forum, 65 (2002), 128-132.

[6] N. Kehayopulu, M. Tsingelis, Fuzzy bi-ideals in ordered semigroups, Information Sciences, 171 (2005), 13-28.

[7] N. Kuroki, On fuzzy ideals and fuzzy bi-ideals in semigroups, Fuzzy Sets and Systems, 5 (1981), 203-215.

[8] N. Kuroki, On fuzzy Semigroups, Information Sciences, 53 (1991), 203236.

[9] N. Kuruki, Fuzzy semiprime quasi-ideals in semigroups, Inform. Sci., 75, No. 3 (1993), 201-211.

[10] S.K. Majumder, On $Q$-fuzzy ideals in $\Gamma$-semigroups, World Academy of Science, Engineering of Technology, 60 (2011), 1443-1447.

[11] A. Rosenfeld, Fuzzy groups, J. Math. Anal. Appl., 35 (1971), 512-517.

[12] X.Y. Xie, Fuzzy ideal extensions of semigroups, Soochow Journal of Mathematics, 27, No. 2 (2001), 125-138.

[13] X.Y. Xie, J. Tang, Regular ordered semigroups and intra-regular ordered semigroups in terms of fuzzy subsets, Iranian Journal of Fuzzy System, 7, No. 2 (2010), 121-140.

[14] L.A. Zadeh, Fuzzy Sets, Information and Control, 8 (1965), 338-353. 
